\title{
PREVALENCE OF TUBERCULOSIS AMONG HEALTHCARE WORKERS IN CAIRO UNIVERSITY HOSPITALS
}

\author{
By \\ ${ }^{1}$ Fouad MM, ${ }^{1}$ Helal SF, ${ }^{1}$ Lotfy BM, ${ }^{2}$ El Kholy AA, ${ }^{3}$ Sabri YY \\ ${ }^{1}$ Occupational and Environmental Medicine, ${ }^{2}$ Clinical Pathology, \\ ${ }^{3}$ Radiology Departments, Faculty of Medicine, Cairo University, Egypt.
}

\begin{abstract}
:
Introduction: Tuberculosis (TB) presents globally as a significant health problem and health care workers $(\mathrm{HCW})$ are at increased risk of contracting TB infection. There is no diagnostic gold standard for latent TB infection (LTBI). Tuberculin Skin Test (TST) is the traditional testing method for diagnosing LTBI, but it has a known high rate of false positive with subsequent needless loss of time, efforts, loss of productivity and side effects. Quantiferon test (QFT) has been developed as a potential replacement for the TST. Aim of work: To assess the prevalence of TB among health care workers in high, medium and low risk areas for TB infection at Cairo University Hospitals (CUH) and to validate the use of Quantiferon as a screening test for TB compared to tuberculin test. Materials and Methods: A cross sectional study with convenient sample of $200 \mathrm{HCWs}$ employed in Cairo University Hospitals and agreed to participate was performed. Information on gender, age, workplace, job title, BCG vaccination and history of both TB and TST were collected using a questionnaire. All participants were subjected to clinical examination, TST, Quantiferon test and chest X-ray. Results: The participants were 70 males and 130 females with mean age + SD $(34.7 \pm 12.6$ years $)$. TST was positive in 86 cases (43\%) and the QFT results were positive in 38 cases (19\%). There was average agreement between the two tests $(76 \%, \mathrm{~K}=0.474)$, concordance between two tests improved from $(\mathrm{\kappa}=0.474)$ at cut-off point $10 \mathrm{~mm}$ to $(\mathrm{\kappa}=0.492)$ at cut-off point $15 \mathrm{~mm}$. LTBI was most common principally among nurses, workers in Chest Department, Internal Medicine Department and technicians in bacteriology lab with highest mean diameter of induration for the studied population in the chest department $(16.6 \mathrm{~mm})$. According to the nature of work the laboratory technicians had the highest mean diameter of induration $(16.58 \mathrm{~mm})$. Thirty-four $(17 \%)$ of the studied
\end{abstract}


population had abnormal chest radiographs suspected of TB. Occupational exposure in high risk area and working duration $>10$ years were statistically significant risk factors for both QFT \& TST, while age and nature of work were not statistically significant risk factors for both tests using multivariable logistic regression. Conclusion and Recommendations: There is risk of tuberculosis transmission among HCWs at Cairo University Hospitals, principally among nurses, workers in Chest Department, Internal Medicine Department and technicians in bacteriology lab. Large scale of screening involving all University hospitals staff must be performed.

We recommend screening of HCWs using TST then confirming positive cases by Quantiferon and following them. Disease probability in HCWs tested positive by serial testing should be further assessed by chest radiograph. Further reinforcement of the infection control policy is a must and treatment regimen for latent TB infection has to be followed.

Key words: Latent tuberculosis infection, health care workers, tuberculin skin test and Quantiferon test.

\section{Introduction}

The risk of transmission of Mycobacterium Tuberculosis from individuals with tuberculosis (TB) to other patients and to health-care workers (HCWs) has been recognized for many years (Fennelly \& Iseman, 1999).

This risk is greater when larger numbers of infectious (smear-positive) TB patients are managed at a healthcare facility, and can be reduced with implementation of effective infectioncontrol measures (Jensen et al., 2005).

In the United States and other highincome countries, the risk of nosocomial transmission of TB was high in the prechemotherapy era, but declined with the reduction in incidence of TB disease in the population (Harries et al., 1997).
In Egypt $\mathrm{TB}$ is considered the second most important public health problem after bilhariziasis (Zaher et al., 2003).

TB is preventable and, in most cases, treatable. Till recently, the 100 years old tuberculin test has been the only available screening test (Zaher et al., 2003). However, tuberculin test does not differentiate between infection and immunological reaction after BCG vaccination.

A new test, Quantiferon-TB Gold (QFT-G, Cellestis Limited, Carnegie, Victoria, Australia), received final approval from the U.S. Food and Drug Administration as an aid for diagnosis of TB infection. This test detects the release of interferon-gamma (IFN-g) 
in fresh heparinized whole blood from sensitized persons when it is incubated with mixtures of synthetic peptides representing two proteins present in $\mathrm{M}$. tuberculosis: early secretory antigenic target-6 (ESAT-6) and culture filtrate protein-10 (CFP-10). These antigens impart greater specificity than is possible with tests using purified protein derivative as TB antigen (CDC, 2003).

Previous study carried at Cairo University Hospitals among health care workers in 2007 revealed that $12 \%$ of the studied population was tuberculin positive, updating this study with Quantiferon test is necessary for proper detection of tuberculosis patients (Abdel Maqsouad et al., 2007).

\section{Aim of work}

To assess the prevalence of $\mathrm{TB}$ among health care workers in high, medium and low risk areas for TB infection at Cairo University Hospitals using Quantiferon as screening test for TB compared to tuberculin skin test.

\section{Materials and Methods}

Study design: A cross sectional study of a convenient sample conducted at Cairo University Hospitals.
Population and sample: Twohundred HCWs (130 females and 70 males) were included in the study in about 4 months during the years 20122013 from the following departments: Chest department, Chest ICU, cardiothoracic ICU and operation room, Bacteriology lab, Operation room (OR), TB Outpatient, Pulmonary function tests (PFT) unit, Rheumatology department, Internal medicine department and ICU, Occupational medicine department, Tropical department, Neurology department, Family medicine department, Psychiatry department, Toxicology centre and Emergency Room (ER) who agreed to participate in this study.

The studied group was divided into high, medium and low risk areas according to TB exposure, the classification of "low risk areas" is applied to settings in which persons with $\mathrm{TB}$ disease are not expected to be present, and, therefore, exposure to M. tuberculosis is unlikely. The classification of "medium risk areas" is applied to settings in which the risk assessment has determined that HCWs will possibly be exposed to persons with TB disease or to clinical specimens that 
might contain M. tuberculosis. "High risk areas" is applied to any setting (or group of HCWs) if evidence suggestive of personto-person (e.g., patient-topatient, patient-to-HCW, HCWtopatient, or HCW to-HCW) transmission of $\mathrm{M}$. tuberculosis has occurred in the setting during the preceding year (CDC, 2005).

All of them were subjected to:

- A questionnaire stressing on workplace, the duration of work, number of previous BCG vaccinations and past tuberculosis history.

- Clinical examination.

- Tuberculin skin test

- Quantiferon-TB Gold test

- Chest X-ray.

\section{Statistical analysis}

Data were statistically described in terms of mean \pm standard deviation $(\mathrm{X} \pm \mathrm{SD})$ and range, frequencies (number of cases) and percentages when appropriate. For comparing categorical data, Chi square $(\chi 2)$ test was performed. Fisher's exact test was used instead when the expected frequency is less than 5. Comparing matched data was done using McNemar test while agreement was tested using kappa statistic. Multivariate analysis was performed using logistic regression modeling with outcomes of TST positivity and QFT-G positivity.

All statistical calculations were done using SPSS version 15 (Statistical Package for the Social Science; SPSS Inc., Chicago, IL, USA) for Microsoft Windows.

Ethical considerations: The study protocol was approved by Occupational and Environmental Department Ethical Committee, Faculty of Medicine, Cairo University. Each participant was explained about the study procedures, objectives and volunteer gave a verbal consent.

Results: Two hundred health care workers, seventy males (35\%) and 130 females $(65 \%)$ were included in this study. Ninety participants $(45 \%)$ worked in high risk areas while 67 $(33.5 \%)$ worked in medium risk areas and $43(21.5 \%)$ worked in low risk areas. Ninety-six health care workers (48\%) are nurses representing the main occupation in the studied subjects, 53 $(26.5 \%)$ are workers, $28(14 \%)$ are doctors, $15(7.5 \%)$ are lab technicians, 
$8(4 \%)$ are administrative personnel dealing with the patients (nutritionist, nurse of pharmacy, pharmacist, secretary and technician of devices). The mean age of the participants + SD was $34.7 \pm 12.6$ years and the mean duration of work + SD (7.824 \pm 8.6209).

All participants had history of previous BCG vaccine since childhood. Two of them (1\%) had booster dose of BCG vaccine in the past. Twenty-five (12.5\%) participants had history of prior TST in the past three years. Nine $(4.5 \%)$ of them underwent conversion of the TST from negative to positive, while $13(6.5 \%)$ of them reported a prior positive TST. Three participants (1.5\%), with no prior history of TST, had 2-step tuberculin test and became TST positive.

Two participants (1\%) had history of previous treatment for active $\mathrm{TB}$ and none had prophylactic treatment to LTBI, only one health care worker $(0.5 \%)$ developed active TB during the study period and was referred to the Chest department to receive treatment. Ninety percent of the HCW reported not using the infection control equipments during contact with TB patients or infectious material, while $10 \%$ used masks and gloves.
All symptoms (cough, expectorations, orthopnea, paroxysmal nocturnal dyspnea, lower limb edema and chest pain) were statistically insignificant except for dyspnea with $\mathrm{p}$ value 0.048 .

Nineteen (9.5\%) persons were asthmatic, $14(15.6 \%)$ of them found in high risk areas with $\mathrm{p}$ value 0.030 which is statistically significant, when compared to other areas. Eighty-six workers $(43 \%)$ had positive tuberculin skin test ( $\geq 10 \mathrm{~mm}), 43(47.8 \%)$ of them in high risk areas with $\mathrm{p}$ value 0.418 which is statistically insignificant. 38 participants (19\%) had positive Quantiferon test,22 (24.4\%) in high risk areas with $\mathrm{p}$ value 0.193 which is statistically insignificant.

The range of induration was (10-27) with highest mean + SD $(16.58 \pm 5.616)$ in lab technicians, while in departments the highest mean+ SD induration was in chest department (16.60土 5.602) with range (10-25).

There was statistically significant correlation between prevalence of positive results regarding both tuberculin and quantiferon tests with duration of work 
Table (1): Relation between tuberculin and Quantiferon results according to nature of work

\begin{tabular}{|c|c|c|c|c|c|c|c|c|}
\hline \multirow{2}{*}{ Nature of work } & \multicolumn{2}{|c|}{ +ve TST } & \multirow{2}{*}{$\mathbf{X}^{2}$} & \multirow{2}{*}{$\mathbf{P}$} & \multicolumn{2}{|c|}{ +ve QFT-G } & \multirow{2}{*}{$\mathbf{X}^{2}$} & \multirow{2}{*}{$\mathbf{P}$} \\
\hline & $\mathbf{N}$ & $\%$ & & & $\mathbf{N}$ & $\%$ & & \\
\hline Nurses (96) & 42 & 43.8 & \multirow{6}{*}{10.48} & \multirow{6}{*}{0.063} & 16 & 17 & \multirow{6}{*}{4.77} & \multirow{6}{*}{0.445} \\
\hline $\begin{array}{l}\text { Administrative } \\
\text { personnel (8) }\end{array}$ & 3 & 37.5 & & & 3 & 37.5 & & \\
\hline Doctors (28) & 9 & 32.1 & & & 3 & 10.7 & & \\
\hline Lab technicians (15) & 12 & 80 & & & 4 & 26.7 & & \\
\hline Workers (53) & 20 & 37.7 & & & 12 & 22.6 & & \\
\hline Total (200) & 86 & 43 & & & 38 & 19 & & \\
\hline
\end{tabular}

Table (1) shows statistically insignificant difference between positive and negative results of both quantiferon and tuberculin tests according to nature of work. However positive results were most common among nurses. 
Table (2): Relation between tuberculin \& Quantiferon results of the studied population according to different departments

\begin{tabular}{|c|c|c|c|c|c|c|c|c|}
\hline $\begin{array}{l}\text { TST \& } \\
\text { QFT-G } \\
\text { Department }\end{array}$ & \multicolumn{2}{|c|}{ +ve TST } & $\mathbf{X}^{2}$ & $\mathbf{P}$ & \multicolumn{2}{|c|}{$\begin{array}{c}\text { +ve QFT-G } \\
\text { \&TST }\end{array}$} & $\mathbf{X}^{2}$ & $\mathbf{P}$ \\
\hline $\begin{array}{l}\text { High risk area (90): } \\
\text { Chest (24) } \\
\text { Chest ICU(17) } \\
\text { Cardiothoracic ICU (18) } \\
\text { Cardiothoracic O.R.(8) } \\
\text { Bacteriology Lab(16) } \\
\text { TB Outpatient (3) } \\
\text { PFT unit (4) }\end{array}$ & $\begin{array}{r}43 \\
10 \\
6 \\
6 \\
4 \\
13 \\
2 \\
2\end{array}$ & $\begin{array}{l}47.8 \\
41.7 \\
35.3 \\
33.3 \\
50 \\
81.3 \\
66.7 \\
50\end{array}$ & 10.56 & 0.103 & $\begin{array}{r}22 \\
9 \\
2 \\
2 \\
3 \\
4 \\
1 \\
1\end{array}$ & $\begin{array}{c}24.4 \\
37.5 \\
11.8 \\
11.1 \\
37.5 \\
25 \\
33.3 \\
25\end{array}$ & 6.30 & 0.391 \\
\hline $\begin{array}{l}\text { Medium risk area (67): } \\
\text { Rheumatology(14) } \\
\text { Internal medicine (23) } \\
\text { Internal medicine ICU } \\
\text { (17) } \\
\text { Occupational medicine } \\
\text { (12) } \\
\text { Tropical (1) }\end{array}$ & $\begin{array}{r}25 \\
3 \\
7\end{array}$ & $\begin{array}{l}52.9 \\
41.7 \\
100\end{array}$ & 5.53 & 0.237 & $\begin{array}{l}2 \\
2 \\
0.0\end{array}$ & $\begin{array}{r}13.4 \\
0.0 \\
21.7 \\
11.8 \\
\\
16.7 \\
0.0\end{array}$ & 3.84 & 0.428 \\
\hline $\begin{array}{l}\text { Low risk area (43): } \\
\text { Neurology }(1) \\
\text { Family medicine }(1) \\
\text { Psychiatry }(23) \\
\text { Toxicology centre }(16) \\
\text { ER(2) }\end{array}$ & $\begin{array}{c}18 \\
1 \\
0.0 \\
13 \\
4 \\
0.0\end{array}$ & $\begin{array}{c}41.9 \\
100 \\
0.0 \\
56.5 \\
25 \\
0.0\end{array}$ & 7.45 & 0.114 & $\begin{array}{l}7 \\
0.0 \\
0.0 \\
5 \\
2 \\
0.0\end{array}$ & $\begin{array}{r}16.3 \\
0.0 \\
0.0 \\
21.7 \\
12.5 \\
0.0\end{array}$ & 1.45 & 0.836 \\
\hline Total HCW (200) & 86 & 43 & & & 38 & 19 & & \\
\hline
\end{tabular}

N.B. HCWs who were Quantiferon positive were tuberculin positive as well.

PFT: pulmonary function test, OR: operation room, ER: emergency room.

Table (2) shows statistically insignificant difference between positive and negative tuberculin results in the studied areas, however the departments with positive tuberculin were the bacteriology lab $13(81.3 \%)$ followed by the psychiatry $13(56.5 \%)$ then the chest $10(41.7 \%)$. It also shows statistically insignificant difference between positive and negative Quantiferon results in different risk areas. Highest percentage of workers with positive Quantiferon were $37.5 \%, 21.7 \%, 21.7 \%$ and $25 \%$ in chest, internal medicine, psychiatry departments then the bacteriology lab respectively. 
Table (3): Frequency distribution of x-ray findings suggestive of Pulmonary Tuberculosis according to tuberculin and Quantiferon positive results.

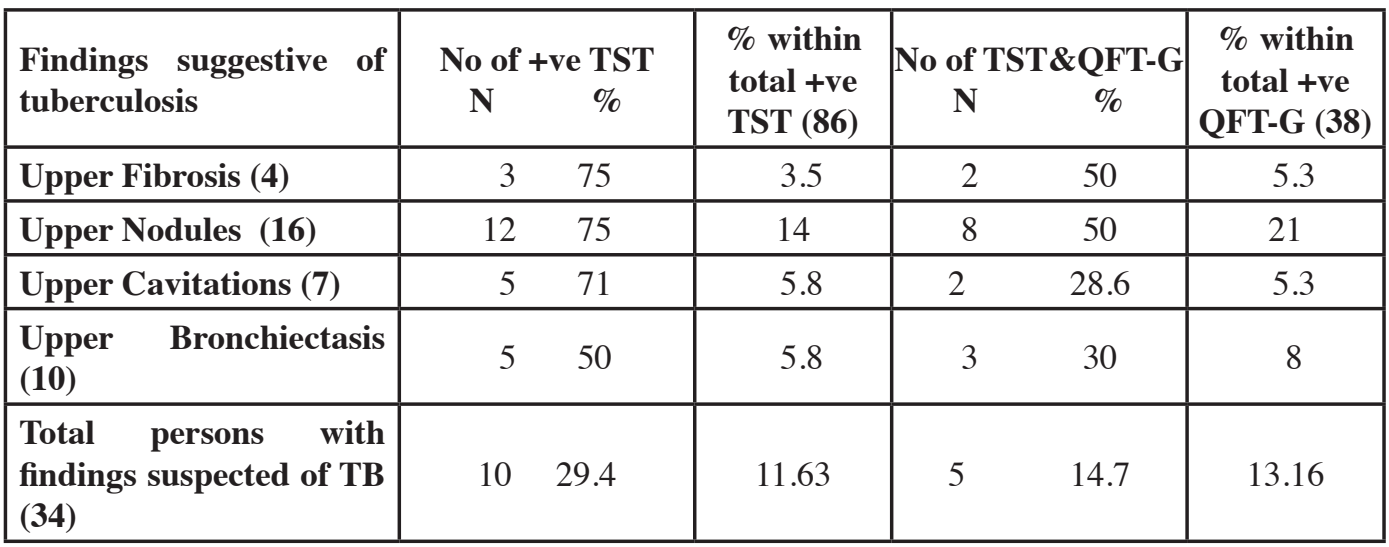

Table (3) shows the frequency distribution of $\mathrm{X}$ - ray findings suggestive of tuberculosis among tuberculin and Quantiferon positive HCWs. Nodules had the highest percentage in both tuberculin and Quantiferon positive cases.

Table (4): Concordance between TST (cut off $10 \mathrm{~mm}$ ) and QFT results

\begin{tabular}{|l|l|c|c|c|}
\hline \multicolumn{2}{|l|}{ QFT-G } & QFT-G (-ve) & QFT-G (+ve) & Total \\
\hline$<\mathbf{1 0}$ (-ve) & Number & 114 & 0.0 & 114 \\
& \% within TST & 100 & 0.0 & 100 \\
& \% within QFT & 70.4 & 0.0 & 57 \\
& \% within total & 57 & 0.0 & 57 \\
\hline$\geq 10$ (+ve) & Number & 48 & 38 & 86 \\
& \% within TST & 55.8 & 44.2 & 100 \\
& \% within QFT & 29.6 & 100 & 43 \\
& \% within total & 24 & 19 & 43 \\
\hline Total & Number & 162 & 38 & 200 \\
& \% within TST & 81 & 19 & 100 \\
& \% within QFT & 100 & 100 & 100 \\
\hline
\end{tabular}

Table (4) shows the analysis of concordance of both QFT and TST results for the criterion of $10 \mathrm{~mm}$ or more ( $\mathrm{P}$ value $<0.001$ ). There is statistical significant agreement (76 $\%)$ with kappa value 0.474 . 
Table (5): Concordance between TST (cut off $15 \mathrm{~mm}$ ) and QFT result

\begin{tabular}{|l|l|c|c|c|}
\hline \multicolumn{2}{|l|}{ QFT in mm } & QFT-G (-ve) & QFT-G (+ve) & Total \\
\hline$<\mathbf{1 5}$ & Number & 149 & 17 & 166 \\
& \% within TST & 89.8 & 10.2 & 100 \\
& \% within QFT & 92 & 44.7 & 83 \\
& \% within total & 74.5 & 8.5 & 83 \\
\hline$\geq 15$ & Number & 13 & 21 & 34 \\
& \% within TST & 38.2 & 61.8 & 100 \\
& \% within QFT & 8 & 55.3 & 17 \\
& \% within total & 6.5 & 10.5 & 17 \\
\hline Total & Number & 162 & 38 & 200 \\
& \% within TST & 81 & 19 & 100 \\
& \% within QFT & 100 & 100 & 100 \\
\hline
\end{tabular}

Table (5) shows the analysis of concordance of the QFT and the TST results for the criterion of $15 \mathrm{~mm}$ or more ( $\mathrm{P}$ value $<0.001)$, with kappa value 0.492 which is considered average agreement. The concordance between the TST and the QFT test in this study was average at cut off point of positive tuberculin test of $10 \mathrm{~mm}$ or more $(\mathrm{k} 0.474)$, and was a little better when cut-off value of positive tuberculin was $15 \mathrm{~mm}$ (k 0.492).

Table (6): Association between risk factors and positive results of QFT and tuberculin

\begin{tabular}{|c|c|c|c|c|c|c|c|c|}
\hline & \multirow[t]{2}{*}{$\begin{array}{l}\text { P value } \\
\text { of QFT }\end{array}$} & \multirow[t]{2}{*}{$\begin{array}{l}\text { OR of } \\
\text { QFT }\end{array}$} & \multicolumn{2}{|c|}{$\begin{array}{c}\text { 95\% CI of } \\
\text { QFT }\end{array}$} & \multirow[t]{2}{*}{$\begin{array}{l}P \text { value of } \\
\text { tuberculin }\end{array}$} & \multirow[t]{2}{*}{$\begin{array}{c}\text { OR of } \\
\text { tuberculin }\end{array}$} & \multicolumn{2}{|c|}{$\begin{array}{l}95 \% \text { CI of } \\
\text { tuberculin }\end{array}$} \\
\hline & & & lower & upper & & & lower & Upper \\
\hline Age & 0.395 & 0.986 & 0.953 & 1.019 & 0.960 & 1.001 & 0.975 & 1.027 \\
\hline Duration of work & 0.000 & 1.092 & 1.042 & 1.143 & 0.001 & 1.077 & 1.032 & 1.125 \\
\hline Occupational exposure & 0.003 & 1.041 & 1.011 & 1.101 & 0.046 & 1.023 & 1.004 & 1.029 \\
\hline Nature of work & 0.434 & 0.958 & 0.860 & 1.067 & 0.522 & 0.972 & 0.892 & 1.060 \\
\hline
\end{tabular}

N.B. OR: Odds Ratio, CI: Confidence Interval.

Table (6) shows multivariate analysis of the risk factors: (age, duration of work, nature of work and occupational exposure in high risk) in relation to tuberculin and Quantiferon results. The duration of work and occupational exposure in high risk areas have statistically significant $p$ value for both Quantiferon and tuberculin tests; while age and nature of work are statistically insignificant. 


\section{List of figures: X-ray findings}

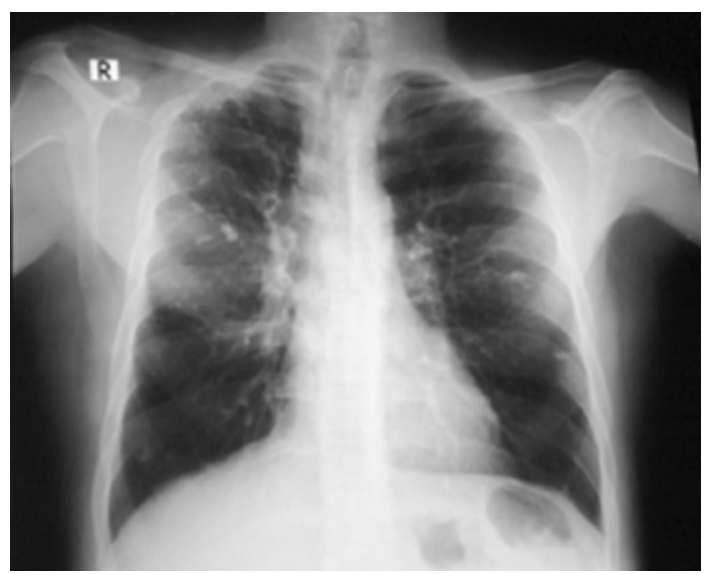

Fig (1): Chest $\mathrm{x}$-ray of 39-year-old male with

LTBI: The right lung field has apical pleural thickening, relative decrease lung volume, calcified nodules and apical cavitities. The Lt lung zone shows mid zonal bronchiectatic changes and there is also bilateral hilar distortion due to fibrosis

Fig (2): Chest x-ray of 57-year- old male with LTBI: Dispersed nodules in both lung fields, peribronchial thickening, unfolded aorta and Lt ventricular configuration of the heart.
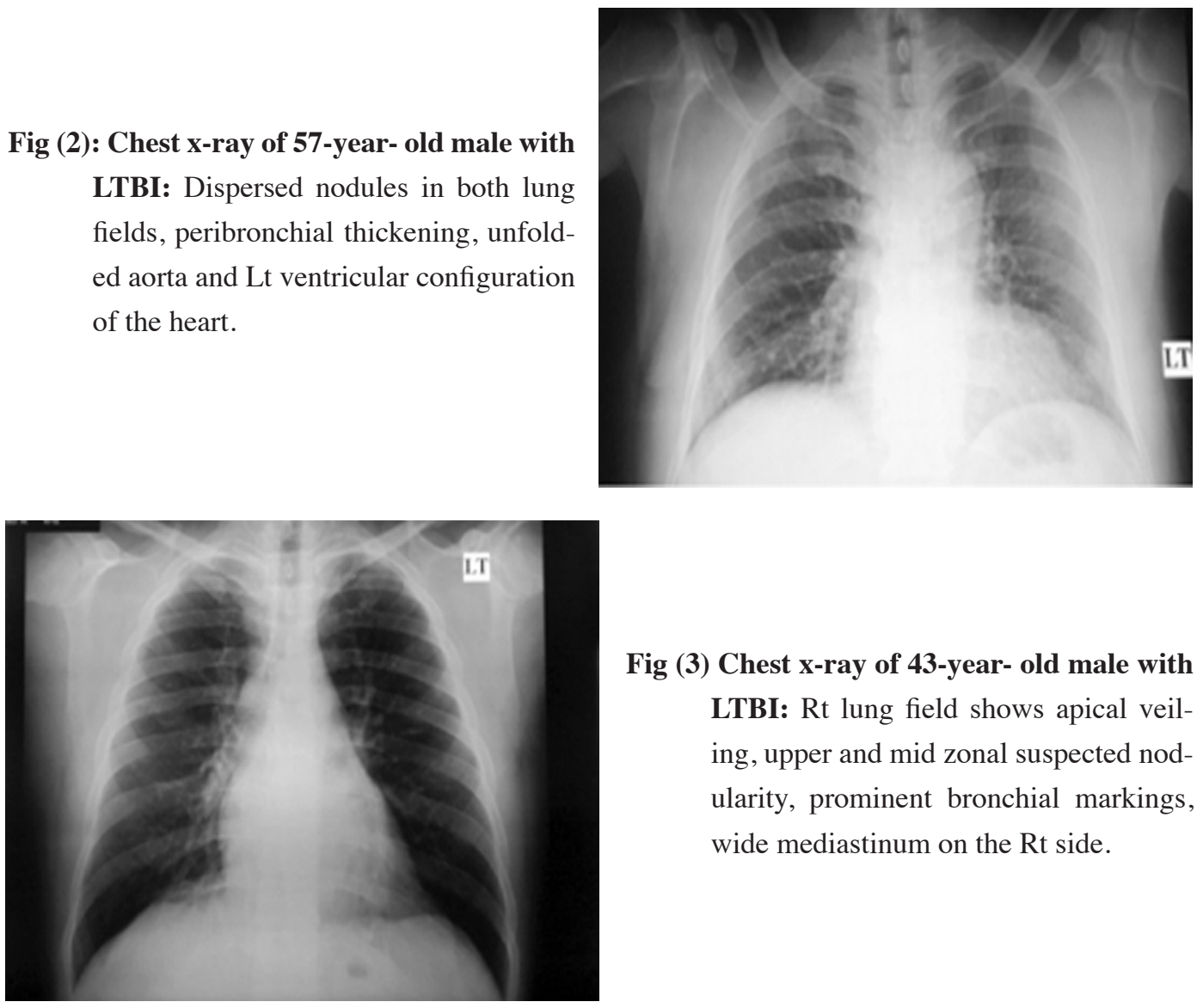

Fig (3) Chest x-ray of 43-year- old male with

LTBI: Rt lung field shows apical veiling, upper and mid zonal suspected nodularity, prominent bronchial markings, wide mediastinum on the Rt side. 
Fig (4) chest x-ray of 17-year-old female with LTBI: Bilateral bronchiectatic changes mostly upper, peribronchial thickening and obliterated waist.
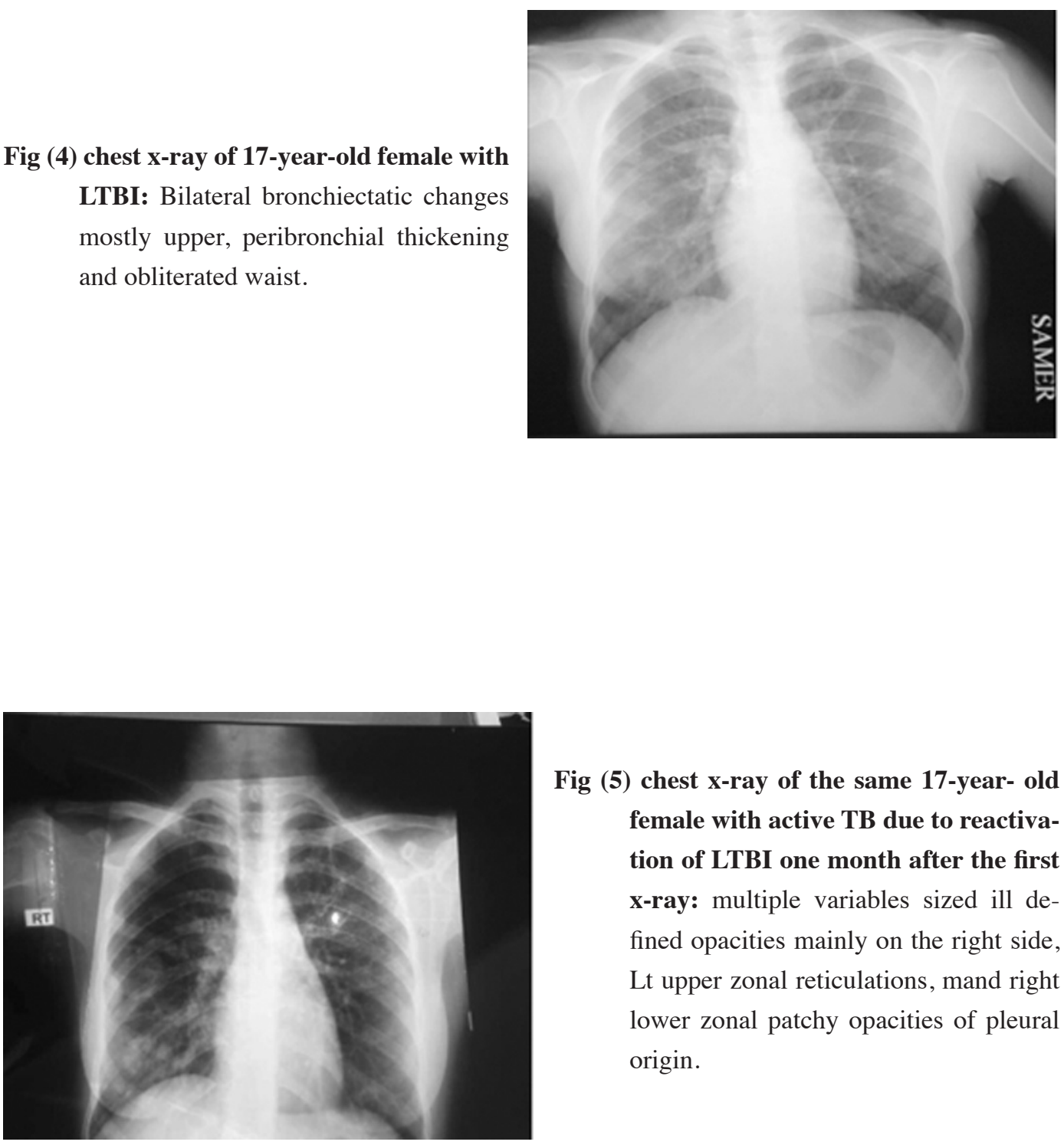

Fig (5) chest x-ray of the same 17-year- old female with active TB due to reactivation of LTBI one month after the first x-ray: multiple variables sized ill defined opacities mainly on the right side, Lt upper zonal reticulations, mand right lower zonal patchy opacities of pleural origin. 


\section{Discussion}

The current study presents a cross sectional study with a convenient sample screening of 200 health care workers at Cairo University Hospitals.

Table (1) shows that only 19\% (n $=38$ ) of the HCW tested positive with QFT in contrast to $43 \%(\mathrm{n}=86)$ tested positive with TST. Nienhaus and his co -workers (2008) investigated 261 HCWs from different types of hospitals who are routinely screened for TB as stipulated by German legislation using QFT and TST following the German Guidelines with a cut off $>5 \mathrm{~mm}$. LTBI prevalence assessed by QFT was $9.6 \%$ compared to $24.1 \%$ with TST which are less than the percentage found by Quantiferon (19\%) and by TST (43\%) in our study as these countries are of low-incidence tuberculosis compared to ours despite that our sample size was larger and our cut off limit was $\geq 10 \mathrm{~mm}$.

In a study from Russia, a TB high endemic country, $47 \%$ of the HCW in the TB department were QFT positive (Drobniewski et al., 2007).

It also shows tuberculin and Quantiferon results among the studied population which were highest among nurses, tuberculin positive test $(n=42)$ and Quantiferon positive test $(n=16)$ while lab technicians had the highest mean diameter of induration $16.58 \mathrm{~mm}$. Tan and his colleagues (2002) agreed with the current study in that nurses in medical wards with long duration of exposure are at risk of developing LTBI as nurses had longer duration of exposure and more frequent exposure to $\mathrm{TB}$ patients taking into consideration that these patients had long stay in-hospital until they are diagnosed as having TB, while in contrast to the current study, Demkow and his co-workers (2008) found that the highest risk of acquiring LTBI disease was associated with TB lab workers (prevalence 50\%) followed by TB ward clinicians (34\%) then nurses $(30 \%)$.

In the current study we found that participants in the high risk areas had the highest percentage in both positive TST $(n=43,47.8 \%)$ and positive Quantiferon test $(\mathrm{n}=22,24.4 \%)$ as shown in table (2). A higher risk of acquiring TB disease was associated with certain work locations as the Bacteriology Lab, the Chest department and the Internal Medicine departments with highest mean diameter of induration 
for participants in the Chest department $(16.60 \mathrm{~mm})$. HCWs in these places do not use respiratory protection when performing high risk procedures such as suctioning or handling specimens whether blood or sputum or during drug administration through nebulizer machine and these procedures are dangerous and can easily transmit TB to HCWs. In contrast to the current study Costa and his co-workers (2010) found ssurprisingly that, the higher the assumed risk of infection the lower was the proportion of TST+ve/QFT+ve concordance where in low risk areas TST was positive in $86.1 \%(n=192)$ of the studied population and Quantiferon was positive in $43.9 \%(n=98)$ while in high risk areas TST was positive in $75.4 \%$ (n $=628$ ) and Quantiferon was positive in $30.7 \%$ ( $\mathrm{n}=256$ ). Whitaker and his coworkers (2013) found a high prevalence of LTBI among Georgian healthcare workers, which was significantly higher among HCWs at TB facilities (55\% QFT positive and 69\% TST positive) compared to HCWs at non-TB healthcare facilities (31\% QFT positive and $54 \%$ TST positive) and these results agreed with the current study. Additionally, alarmingly high rates of LTBI diagnostic test conversions were found among Georgian HCWs working at a TB facility.

In the current study all participants had a chest X-ray (CXR) and it was found that $34 / 200(17 \%)$ of all the HCWs have abnormal chest radiographs suspected of TB .Sixteen (8\%) of them have upper nodular shadows, $4(2 \%)$ had upper fibrotic lesions, 7 (3.5\%) had upper cavitations and $10(5 \%)$ had upper lobar bronchiectasis. Ten participants out of the 34 were tuberculin positive and 5/34 were Quantiferon positive as shown in table (3). In accordance with the current study, Gracía-Gasalla and his co-workers (2013) found that 7 patients out of 123 had a CXR suggestive of past TB, 3 had a positive TST, and 2 had a positive QFT, while in contrary to the current study, Gran and his co-workers (2013) did not find any radiological signs of active TB in any of the HCW. Persons with LTBI typically have normal chest radiograph, although they might have abnormalities suggestive of previous $\mathrm{TB}$ disease or other pulmonary conditions. Previous healed TB disease typically produces radiographic findings that differ from those associated with current TB disease. These findings include nodules, 
fibrotic scars, calcified granulomas, and apical pleural thickening (CDC, 2006).

The prevalence of LTBI varied widely depending on the test used. Therefore Torres and his co-workers (2009) estimated that restricting radiographs to symptomatic or QFT -positive HCWs would have reduced the number of radiographs by more than half (TST 74.2\% versus QFT 32.6\%) without decreasing the number of active TB cases detected in this population, they also estimated that the increase of TST diameter in the small subgroup (n =59) tested three times during the study period most likely indicates a booster effect of serial testing as does the higher rate of TST+/QFT- results in nurses, who also have TST repeated most often. The effect of serial testing with TST on QFT has still to be elucidated. Preliminary results suggest that QFT was not substantially influenced when TST is administered 3 days before the QFT (Leyten et al., 2007).

The results of a study by Kuś and his co-workers (2011) showed that there is a higher consistency with QFT test results for positive TST results of $15 \mathrm{~mm}$ than for $10 \mathrm{~mm}$ and confirmed that the $15-\mathrm{mm}$ cut-off value is more specific. This somewhat coincides with our results where we found a concordance of $76 \%$ (at TST $>10 \mathrm{~mm}$ ) between TST and QTF with kappa = 0.474 which increases a little at cut-off point $\geq 15 \mathrm{~mm}$ with $\mathrm{kappa}=0.492 \mathrm{as}$ shown in tables $(4 \& 5)$.

Results from the multivariable analysis assessing the association between LTBI risk factors and TST positivity were consistent with the study of Zwerling and his colleagues (2012), where total years worked in health care has been found to be consistently associated with TST positivity and this agreed with the results of our study. In the current study, we did not find any effect of the age on the QFT results or the TST results as shown in table (6). In accordance with our findings the study of Gran and his co-workers (2013) didn't also find any effect of age on Quantiferon results.

In contrast to the current study in a multivariate analyses by Jo and his co-workers (2013) for age, gender, nature of work, experience of working in TB-related departments and degree of contact with TB patients, only age was independently associated with increased risk of a positive TST result, 
while age and experience of working in TB-related departments (OR, 2.42, 95\% CI, 1.10-5.34) were independently associated with increased risk of having a positive QFT result.

The data indicates that a positive QFT correlate with a high progression rate but the number of cases is still small and these results need to be interpreted with care. The study from Diel and his co-workers (2008) on disease probability after positive QFT showed that, out of 41 participants with a positive QFT results, 14.6\% developed TB within the 103 weeks of observation. The progression rate for TST-positives was only $2.3 \%$. Thus the progression rate estimated by QFT was higher than the one estimated by WHO (2006) for lifetime after positive TST (5 to 10\%). Many studies conclude that due to the high negative predictive value, progression to active $\mathrm{TB}$ in healthy immune competent individuals with negative QFT is very unlikely. Use of QFT in this population is expected to increase diagnostic specificity and improve acceptance of treatment for LTBI (Nienhaus et al., 2011). In the current study we did not follow the patients for long period however during the study period only $1(0.5 \%)$ which was TST and Quantiferon positive, converted to active disease within 3 weeks of the diagnosis of LTBI, however she had history of active TB a year prior to the study and she received treatment for 6 months.

In the current study we found that only $19 \%$ of the HCW were positive with QFT in contrast to $43 \%$ with TST, by this it is obvious that TST generally is of little usefulness. Instead, QFT offers better specificity and thereby lower prevalence of positive tests and fewer HCW who require X-rays, further clinical follow-up or LTBI treatment. Still, medical follow-up of HCW with strong TST reactions and risk factors for TB reactivation might be warranted since there is no diagnostic gold standard for LTBI.

The limitations of our study are the small number of QFT positive to draw strong conclusions on the overall prevalence of $\mathrm{TB}$ infection in $\mathrm{HCW}$ in Cairo University Hospitals, the risk factors for $\mathrm{TB}$ infection and the effects of serial testing. In addition it is not possible to show the frequency of converters since no repetitive testing of the QFT negative were performed as 
it is the first time in Cairo University Hospitals to use Quantiferon test as a screening test.

\section{Conclusion and Recommendation}

There is risk of tuberculosis transmission among $\mathrm{HCWs}$ at Cairo University Hospitals, principally among nurses, workers in Chest Department, Internal Medicine Department and technicians in bacteriology lab. The prevalence of $\mathrm{TB}$ among HCWs was $19 \%$ by Quantiferon and $43 \%$ by TST which is rather high as compared to other low incidence countries for TB. There was moderate agreement between the two tests however all HCWs with negative Quantiferon were negative TST as well. Comparing results of TST and QFT-G it was found that concordance improved from $(x=$ $0.474)$ at cut-off point for induration sizes of $10 \mathrm{~mm}$ or more, to $(x=0.492)$ at cut-off point $15 \mathrm{~mm}$ or more. Risk factors associated with positive results were duration of work and occupational exposure in high risk areas.

We recommend a risk assessment for TB infection needs to be performed. Large scale of screening involving all University hospital staffs especially those in high risk areas should be done at least annually. Establishing a tuberculosis prevention program that must include all health care workers. Pre-placement medical examination must include chest X-ray, TST and Quantiferon for those who are TST positive. Periodic assessment of the staff who is TST positive using Quantiferon. Repeating QFT test to those who are QFT negative in serial testing during periodic examination to show the frequency of converters should be performed. A treatment regimen for latent TB infection has to be followed for those who are tuberculin and Quantiferon positive, the Quantiferon is much more expensive than TST so, we recommend screening of HCWs using TST then confirming those positive TST by Quantiferon and following them up every year using Quantiferon.

\section{Competing interests}

Authors have declared that no competing interests exists.

\section{Acknowledgment}

the authors would like to thank Dr Gehad Abo EL Atta ; Prof of Occupational and Environmental Medicine ; Faculty of medicine, Cairo University, for his great effort in 
facilitating the requirement of this work, also the health care workers who agreed to participate voluntarily in this study .

\section{References}

1. Abdelmaqsoud A, Lotfy B and El Shewy Y T (2007): Assessing the prevalence of tuberculosis in paramedical personnel at Cairo University Hospitals and the impact of liver affection on the treatment decision. Sci Med J; 19(1):67-76.

2. CDC (2003): Guidelines for using the QuantiFERON®-TB test for diagnosing latent Mycobacterium tuberculosis infection. MMWR (52) (No. RR-2): 15-18.

3. CDC (2005): Guidelines for Preventing the Transmission of Mycobacterium tuberculosis in Health Care Settings. MMWR Vol. 54/ No.RR-17; 1-141.

4. CDC (2006): Prevention and control of Tuberculosis in Correctional and Dentition Facilities. MMWR; 55(27):1-44.

5. Costa J, Silva R, Sá R, et al., (2010): Comparison of interferon - release assay and tuberculin test for screening in healthcare workers. Rev Port Pneumol; XVI (2): 211-221.

6. Demkow U, Broniarek-Samson B, Filewska M et al., (2008): Prevalence of latent tuberculosis infection in health care workers in Poland assessed by interferon-gamma whole blood and tuberculin skin tests. Journal of Physiology and Pharmacology; 59(6):209-217.

7. Diel R, Loddenkemper R, Meywald-Walter K, Niemann S and Nienhaus A (2008): Predictive value of a whole-blood IFN-\{gamma\} assay for the development of active TB disease. Am J Respir Crit Care Med; 177:1164-1170.

8. Drobniewski F, Balabanova Y, Zakamova E, Nikolayevskyy V and Fedorin I (2007): Rates of latent tuberculosis in health care staff in Russia. PLoS Med ; 4: e55.

9. Fennelly K and Iseman M (1999): Health care workers and tuberculosis: The battle of a century. Int J Tuberc Lung Dis ;3: 363-364.

10. Gracía-Gasalla M,Fernández-Baca V, Juan-Mas A et al., (2013): Use of Quantiferon-TB-Gold in Tube ${ }^{\circledR}$ test for detecting latent tuberculosis in patients considered as candidates for anti-TNF therapy in routine clinical practice. Enferm Infec Microbiol Clin ; 31(2): 76-81.

11. Gran G, Aßmus J and Ma Dyrhol-Riise A (2013): Screening for latent tuberculosis in Norwegian health care workers: high frequency of discordant tuberculin skin test positive and interferon-gamma release assay negative results. BMC Public Health; 13: 353.

12. Harries A, Maher D and Nunn P (1997): Practical and affordable measures for the protection of health care workers from tuberculosis in lowincome countries. Bull World Health Organ; 75: 477-489.

13. Jensen $\mathrm{P}$, Lambert $\mathrm{L}$, Iademarco $\mathrm{M}$ and Ridzon $R$ (2005): Guidelines for preventing the transmission of Mycobacterium tuberculosis in health care settings. MMWR; 54(RR-17):1141.

14. Jo K, Hong Y, Park J et al., (2013): Prevalence of Latent Tuberculosis Infection among Health Care Workers in South Korea: A Multicenter Study. Tuberc Respir Dis (Seoul); 75(1): 18-24.

15. Kuś J, Demkow U, Lewandowska k, et al., (2011): Prevalence of latent infection with Mycobacterium tuberculosis in Mazowieckie province using interferon gamma release assay after stimulation with specific antigens ESAT6 and CFP-10. Pneumonologiai Alergologia Polska; 79(6): 407-418.

16. Leyten E,Prins C,Bossink A, et al.,(2007): Effect of tuberculin skin testing on a Mycobacterium 
tuberculosis specific interferon- $\gamma$ assay. Eur Respir J; 29: 1212-1216.

17. Nienhaus A, Schablon A, Costa J and Diel $R$ (2011): Systematic review of cost and cost effectiveness of different TB-screening strategies. BMC Heal Serv Res; (11):247.

18. Nienhaus A, Schablon A, Siano B, le Bacle C and Diel R (2008) : Evaluation of the Interferongamma Release Assay in Healthcare Workers. Int Arch Occup Enviro Health; 81: 295-300

19. Tan L, Kamarulzaman A, Lian $\mathrm{C}$ and Lee $T$ (2002): Tuberculin skin testing among healthcare workers in the University of Malaya Medical Centre, Kuala Lumpur, Malaysia. Infect Control Hosp Epidemiol; 23(10):584590.

20. Torres J, Sà R, Cardoso M, Silva R et al., (2009): Tuberculosis screening in Portuguese health care workers using the tuberculin skin test and the interferon- $\gamma$ release assay. Eur Respir J; 34: 1423-1428.

21. Whitaker A, Mirtskhulava V, Kipiani M, et al., (2013): Prevalence and Incidence of Latent Tuberculosis Infection in Georgian Healthcare Workers. PLoS ONE; 8(3): e58202.

22. World Health Organisation (2006): Stop TB Partnership; Global Plan to Stop TB 20062015 - Actions for life, towards a world free of tuberculosis. Int J Tuberc Lung Dis; 10 (3): 240-241.

23. Zaher H, Tag El Din $M$ and Van Maaren $P$ (2003): Tuberculosis Control: The Experience of Egypt. National Tuberculosis Program Report, Egyptian Ministry of Health, Cairo, Egypt.

24. Zwerling A, Cojocariu M, McIntosh F, et al., (2012): TB Screening in Canadian Health Care Workers Using Interferon-Gamma Release Assays. PLoS One; 7(8): e43014. 